\section{Diffuse cutaneous mastocytosis masquerading as linear IgA bullous dermatosis of child- hood}

\author{
Tuntas Rayinda, Dyah Ayu Mira \\ Oktarina, Retno Danarti \\ Department of Dermatology and \\ Venereology, Faculty of Medicine, Public \\ Health and Nursing, Universitas Gadjah \\ Mada, Yogyakarta, Indonesia
}

\begin{abstract}
Diffuse cutaneous mastocytosis is a rare form of cutaneous mastocytosis that can appear in heterogeneous clinical presentations, including eruption of papules, erythematous plaques, blisters, and erythroderma. We report a 1.5year-old boy who presented with itchy wheals and blisters spreading on his body. The patient was initially managed as a linear IgA bullous dermatosis of childhood (LABD) because of the similarity of clinical symptoms and the presenting of linear IgA deposits at the basement membrane. Due to the development of urticarial plaque after the resolution of the blisters, the diagnosis of diffuse cutaneous mastocytosis was made based on clinical, histopathological (hematoxylin-eosin, Giemsa, and toluidine blue staining), and direct immunofluorescent examinations ( $\operatorname{IgA}$, IgG, IgM, C3). The symptoms were improved following antihistamines and oral corticosteroid treatment.
\end{abstract}

\section{Introduction}

Mastocytosis is a group of diseases with clinical symptoms caused by massive infiltration of mast cells in various tissues, including skin, blood, gastrointestinal, cardiovascular, and musculoskeletal systems. ${ }^{1}$ Nettleship first described the disease entity in 1889 as a rare form of urticaria. ${ }^{2}$ Mastocytosis has a prevalence of 1 in 10,000 population, and the incidence ranges from 5 to 10 cases per million individuals per year in the United States. ${ }^{3}$

Cutaneous mastocytosis is the most common form of mastocytosis in children and has several forms, including urticaria pigmentosa, diffuse cutaneous mastocytosis (DCM), mastocytoma, and teleangiectacia macularis eruptive perstans. ${ }^{4,5}$ Diffuse cutaneous mastocytosis is rare form of pediatric mastocytosis, and can manifest as an eruption of papules, erythematous plaques, bullae, and erythroderma followed by skin thickening and pigmentation changes. ${ }^{6}$ The diagnosis of DCM in children, especially those presenting as bullous eruption (namely, diffuse bullous cutaneous mastocytosis), is a challenge for clinicians and symptoms can mimic other bullous eruptions. Other than DCM, the bullous eruption in infants and children can also develop in either acquired or inherited bullous disorders, such as linear IgA bullous dermatosis in childhood (LABD), staphylococcal scalded skin syndrome, juvenile bullous pemphigoid, and epidermolysis bullosa simplex.

We present a DCM case in a child initially managed as LABD because of its similar clinical symptoms and features of linear IgA deposits on the basement membrane in direct immunofluorescence (DIF) examinations. This case report emphasizes the importance of comprehensive clinical, histological and DIF examinations in managing children with acquired bullous eruption. Moreover, clinicians should consider DCM as one of the differential diagnoses of infants or children presenting with bullous lesions.

\section{Case Report}

A 1.5-year-old boy was brought to our center with blisters on his face and body, starting three days before the admission. Six days earlier, the patient had fever, cough, and runny nose. The patient was treated with paracetamol and amoxicillin for two days by a general practitioner (GP). After the fever receded, flaccid blisters developed on his face and body.

In past medical history, the patient had experienced a similar complaint one month before. At that time, the complaint was not treated and he selfimproved within two weeks. The patient also had a history of recurrent
Correspondence: Tuntas Rayinda, Department of Dermatology and Venereology, Faculty of Medicine, Public Health, and Nursing, Universitas Gadjah Mada, Yogyakarta, Indonesia.

E-mail: tuntasrayinda@ugm.ac.id

Key words: cutaneous mastocytosis, bullous mastocytosis, chronic bullous disease of childhood, linear IgA dermatosis of childhood.

Acknowledgements: Authors would like to thank the staff in Klinik Bahasa for the proofreading and language editing.

Contributions: The authors contributed equally.

Conflict of interest: The authors declare no potential conflict of interest.

Funding: None.

Ethics approval: Approved

Consent to publication: Received.

Availability of data and materials: Available from the authors.

Please cite this article as: Rayinda $T$, Oktarina DAM, Danarti R. Diffuse cutaneous mastocytosis masquerading as linear IgA bullous dermatosis of childhood. Dermatol Rep 2021;13:9021.

Received for publication: 21 November 2020. Revision received: 18 January 2021.

Accepted for publication: 20 January 2021.

This work is licensed under a Creative Commons Attribution-NonCommercial 4.0 International License (CC BY-NC 4.0).

(C) Copyright: the Author(s), 2021

Licensee PAGEPress, Italy

Dermatology Reports 2021; 13:9021

doi:10.4081/dr.2021.9021

urticaria with unknown causes. There was no family history with a similar condition.

Dermatological examination revealed tense blisters with clear fluid in various sizes scattered on the face and back. Several skin-colored papules and skin erosions were also found on his body (Figure 1). Nikolsky's sign, Darier sign, and dermographism were negative during examination. Neither lymph node enlargement nor hepatosplenomegaly were found.

Based on clinical examination, the working diagnosis established at that time was LABD, and the therapy given was normal saline $(0.9 \%$ sodium chlo- 
ride) gauze dressing on the top of bullae followed by silver sulfadiazine $1 \%$ cream applied twice daily to areas experiencing erosion. At this appointment, skin biopsy was considered, but the parents refused. Four months later, the patient came back with the appearance of rashes that felt very itchy. The lesions had emerged on the chest and spread to the back and extremities. At this appointment, no new blisters had developed. Dermatology examination revealed wheals and hyperpigmented patches, distributed on the face, chest, back, and abdomen (Figure 1). The patient was still managed as LABD and was given methylprednisolone $4 \mathrm{mg}$ tablets twice daily for seven days and cetirizine $2.5 \mathrm{mg}$ daily.

Two weeks later, the symptoms were improved, but there were still new erythematous wheals on the back, face, and chest. During the examination,

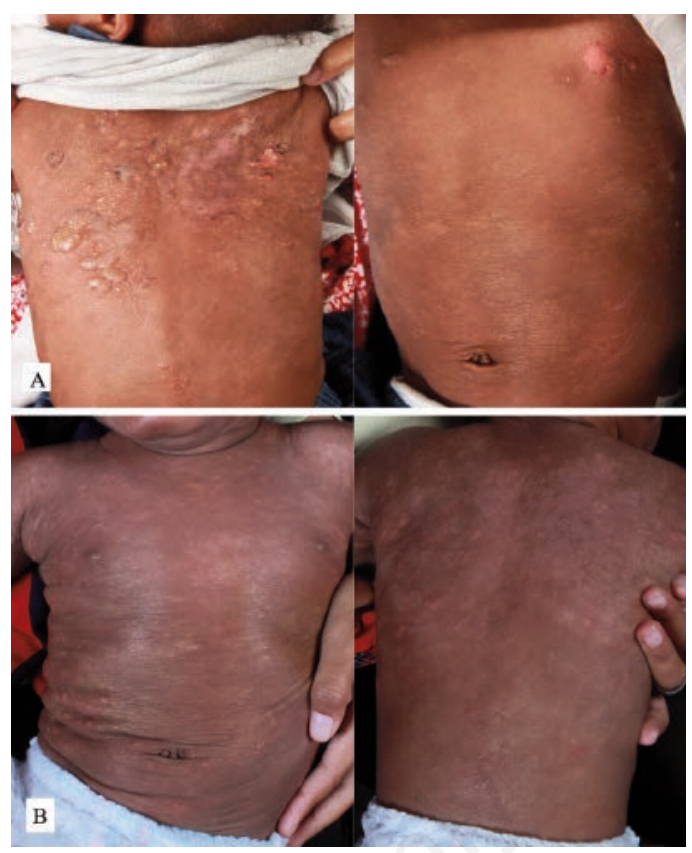

Figure 1. Clinical picture of the patient at initial visit: blisters and erosion distributed on the trunk (A), and the development of new urticarial plaques after blisters resolution (B).

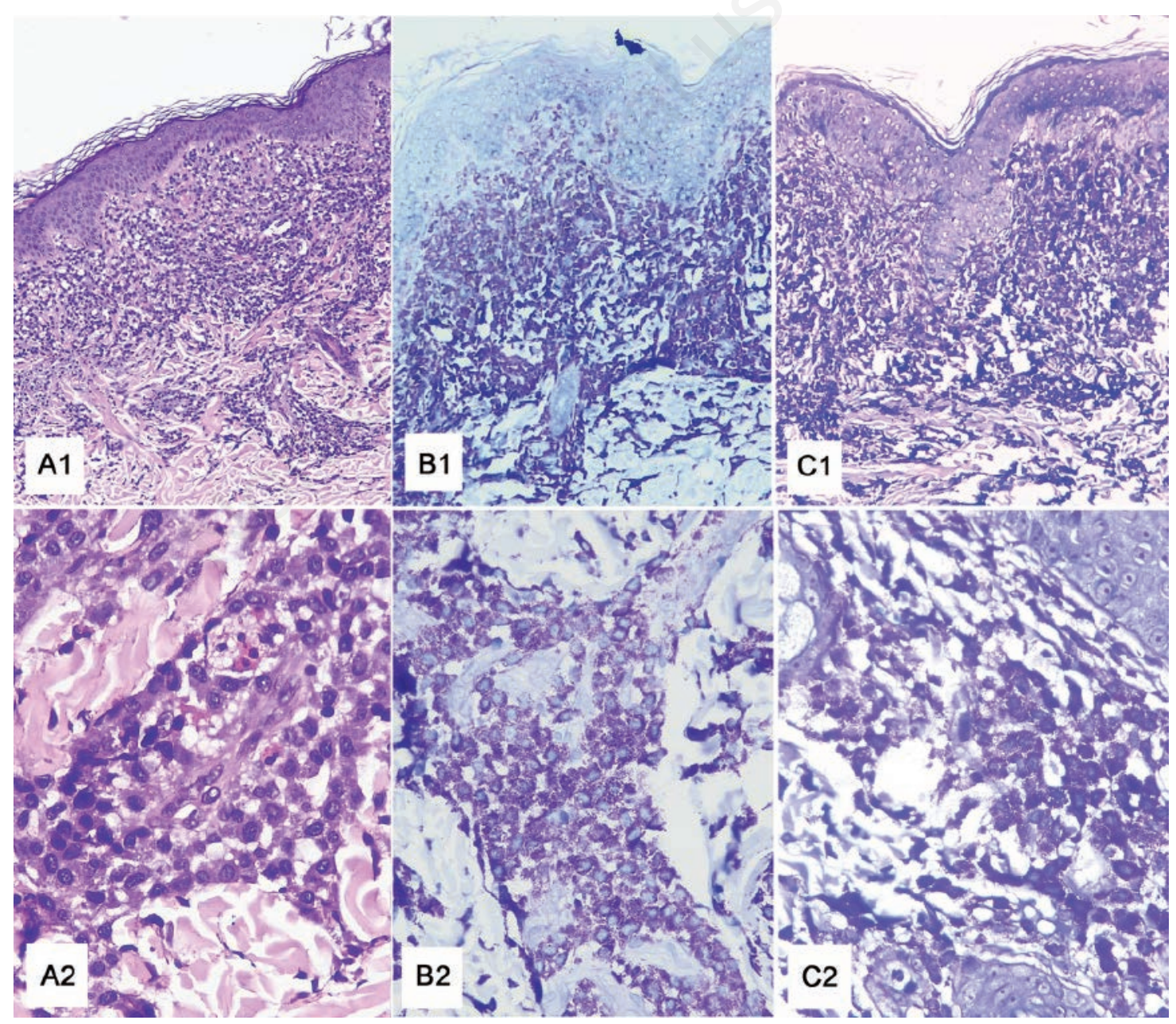

Figure 2. Histopathology staining with hematoxylin-eosin (A1, A2), Toluidine blue (B1, B2), and Giemsa (C1, C2), showed massive infiltration of mast cells in the epidermis and dermis. 
more erythematous patches and wheals were found with hyperpigmented patches distributed on the face, chest, back, and abdomen. There were no signs of Darier nor dermographism. At this point, the parents agreed to give consent for the skin biopsy procedure for the patient.

Histopathological examination with hematoxylin-eosin (HE) staining from urticarial lesions taken from the back area showed basket weave-type orthokeratosis, spongiosis, acanthosis, and elongation of rete-ridge in the epidermis. Infiltration of inflammatory cells, consisting of lymphocytes, a few eosinophils, histiocytes, and a large number of mast cells, was found in the upper dermis, perivascular, and surrounding the skin appendages. Neither a subepidermal cleft nor any neutrophil were seen. Additional staining using Giemsa and toluidine blue showed a large number of mast cells in the dermis layer (Figure 2). These findings support the diagnosis of cutaneous mastocytosis.

DIF examination using $\operatorname{IgA}, \operatorname{Ig}$, $\mathrm{IgG}$, and complement antibodies (C3) was done on the patient's skin tissue taken from the same area and revealed
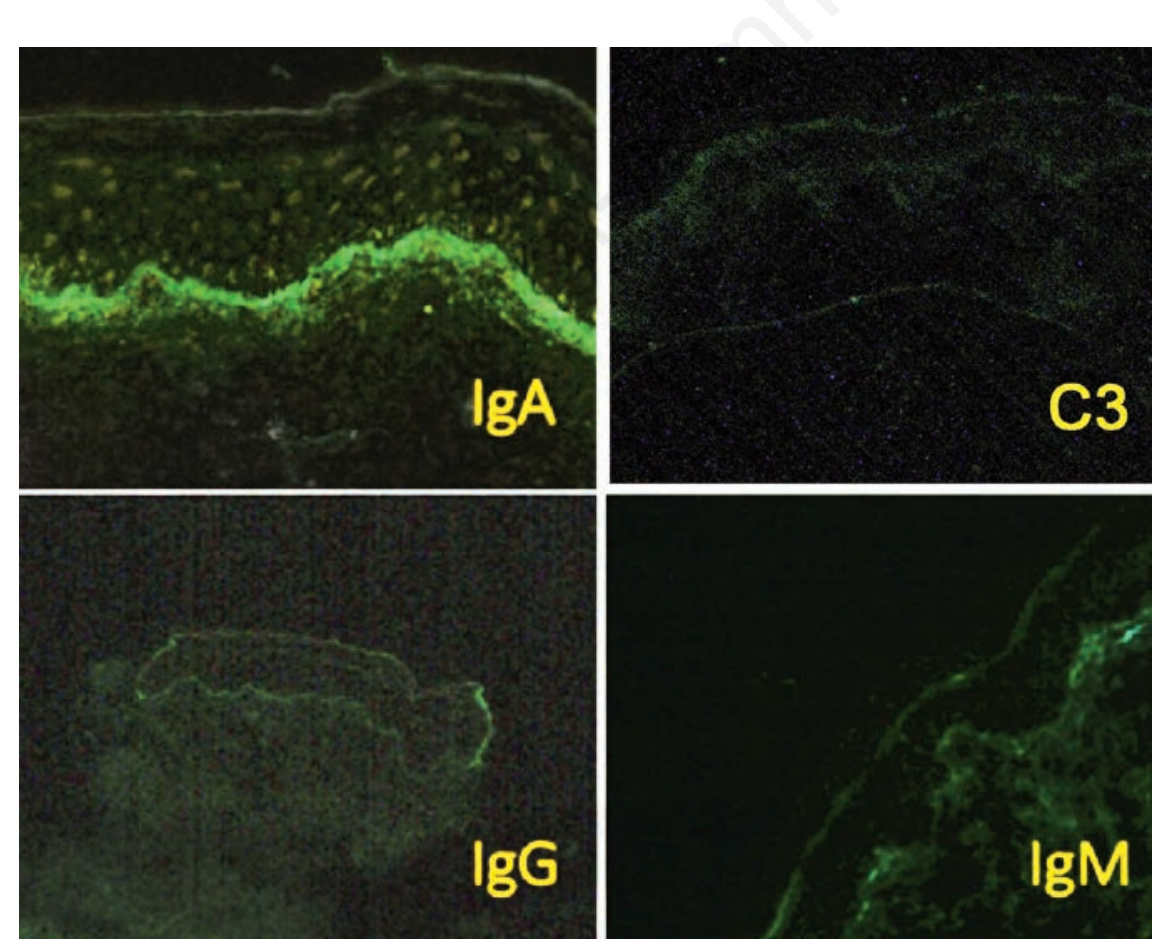

Figure 3. Immunofluorescent staining showed linear Immunoglobulin A (IgA) deposi-

tion in the basement membrane.

linear IgA deposits the basement membrane. The staining for $\operatorname{IgM}, \operatorname{IgG}$, and C3 were negative (Figure 3 ).

Based on the clinical and supporting examination, the diagnosis of cutaneous mastocytosis was made in this patient. The therapy given was cetirizine $2.5 \mathrm{mg}$ per day and topical moisturizer. Two weeks later, there were no new lesions had developed, and the treatment was continued with the application of topical moisturizer twice daily. However, new blisters reappeared one week later. The patient went to a GP and received oral prednisone $2.5 \mathrm{mg}$ per day for one month. Up to two weeks after the patient stopped taking oral prednisone, there was no any sign of relapse.

\section{Discussion and Conclusions}

Cutaneous mastocytosis is a disease caused by the release of mast cell mediators and/or mast cell infiltration in the skin tissue. Physiologically, stem cell factors (SCF) binding to the Kit receptor in the extracellular area activate mast cells. This binding increases the proliferation and extends the lifespan of mature mast cells and triggers the release of mast cells' mediators. In about $60 \%$ to $80 \%$ of mastocytosis cases, somatic mutations of the gene encoding the Kit protein cause autocrine dysregulation and activation of Kit in the absence of the SCF ligand. ${ }^{7,8}$ The excessive activation of Kit protein leads to the involvement of internal organs other than the skin. ${ }^{7}$

Most of the patients with cutaneous mastocytosis do not meet all of the criteria for systemic mastocytosis, in which there is a massive infiltration of mast cell in various organs, including the bone marrow and liver. Although internal organ involvement is rare, cutaneous mastocytosis is often accompanied by gastrointestinal symptoms and anaphylaxis. ${ }^{7}$ We did not find any sign of internal organ involvement in our case.

Skin biopsy is crucial in diagnosing cutaneous mastocytosis. Histopathological examination of DCM reveals a large number of mast cell infiltrations in the perivascular, interstitial, or nodular patterns found in the upper papillary and reticular dermis. ${ }^{9}$ In our case, histopathological examination showed abundant mast cell infiltration, mainly in the upper dermis. Thus, the diagnosis of cutaneous mastocytosis could be confirmed.

The clinical findings in our patient are consistent with DCM and the histopathologic examination supported the diagnosis. However, we did not perform bone marrow analysis, KIT mutation, and serum tryptase to exclude systemic involvement of mastocytosis. Theoharides and colleagues suggested that children presenting with skin lesions typical for DCM without any clinical signs of hepatosplenomegaly and lymphadenopathy do not require bone marrow biopsy and evaluation. ${ }^{7}$ In contrast, mastocytosis in adults is often associated with systemic involvement. Of note, patients with systemic mastocytosis may not show any skin involvement. ${ }^{7,10}$ Lange and colleagues suggested that serum tryptase evaluation is necessary as a consideration before performing bone marrow biopsy in pediatric mastocytosis cases. ${ }^{11}$

At the early onset, we managed our 
patient as LABD due to the similarity of skin lesions. Nevertheless, following the blister resolution, urticarial plaques developed at the same locations as the former lesions. The absence of a subepidermal cleft with infiltration of neutrophilic inflammatory cells and the presence of many mast cell infiltrating in the histopathology examination excluded the diagnosis of LABD.

Linear IgA deposition on the basement membrane is a key finding from direct immunofluorescent examination in LABD. ${ }^{12}$ Various inflammatory dermatoses, such as dermatitis herpetiformis and Henoch-Schoenlein purpu$\mathrm{ra}$, and bullous lupus erythematosus, can also exhibit IgA deposition. Even though the role of IgA in the subepidermal blister formation remains unclear, it is assumed to involve neutrophilic infiltration mediated by $\operatorname{IgA} .{ }^{13}$ Interestingly, in our patient, DIF examination revealed linear deposits of IgA on the basement membrane. Whether the IgA deposition is caused by the inflammatory process in cell mast degranulation of coincidence with LABD remains unknown. Slavescu and colleagues have reported a case of bullous cutaneous mastocytosis accompanied by findings of IgM deposits in the basement membrane. ${ }^{14}$ Nevertheless, until now, there is no reported case of linear $\operatorname{Ig} \mathrm{A}$ deposition on the basement membrane in patients with cutaneous mastocytosis.

The management of cutaneous mastocytosis in children is comprised of non-pharmacological and pharmacological therapies. Non-pharmacological management includes avoiding various triggers such as skin friction, exposure to high temperatures such as hot baths, and vigorous physical activities. Other known precipitating factors include fever, teething, diet, vaccinations, and substances that can trigger mast cell degranulation such as non-steroidal anti-inflammatory drugs, anticholinergics, aspirin, narcotics, polymyxin $\mathrm{B}$ sulfate, and alcohol. ${ }^{1}$

Pharmacological management of pediatric mastocytosis includes topical and systemic therapies. For topical treatment, corticosteroid cream can be used along with the local care of skin with a moisturizer. Treatment with $\mathrm{H}_{1}$ and $\mathrm{H}_{1}$ antihistamines, oral disodium cromolyn, and leukotriene receptor antagonists can be considered as drug choices for severe to moderate pediatric cutaneous mastocytosis. ${ }^{15}$ Oral glucocorticoid is recommended for diffuse cutaneous disease refractory to topical treatment, but the supporting evidence is scarce..$^{16}$ In our case, the symptoms improved after the administration of antihistamines and moisturizer. Oral corticosteroid given by a GP for one month resulted in a good therapeutic response as well.

Cutaneous mastocytosis commonly occurs in early life and can improve during puberty. Generally, this disorder is benign and has a favorable prognosis. ${ }^{17}$ Nevertheless, $15-30 \%$ of cutaneous mastocytosis in children will persist in adults and have systemic involvement. ${ }^{18}$ In our patient, improvement occurred after administration of antihistamines, but complaints returned when the drug was stopped. After receiving oral corticosteroids from a GP for one month, the skin lesions improved and did not recur within two weeks after discontinuation of oral corticosteroids. Antihistamines act as a competitive antagonist on histamine receptors and help the stabilization of mast cell activity. ${ }^{19}$ Whereas, corticosteroids can decrease the number of mast cells in vitro, but they do not have any effect on the release of degranulated mast cells products. ${ }^{20}$

In summary, we report a DCM case of an otherwise healthy 1.5-year-old boy who was initially managed as LABD. The diagnosis of DCM was confirmed by clinical and histopathological examinations using $\mathrm{HE}$, Giemsa, and toluidine blue staining. This case underscores that DCM should be admitted as a differential diagnosis of an acquired bullous eruption in infants or children and can present with bullous eruptions alongside the linear IgA deposition on the basement membrane that imitates LABD.

\section{References}

1.Ben-Amitai D, Metzker A, Cohen HA. Pediatric cutaneous mastocytosis : a review of 180 Patients. Isr
Med Assoc J 2005;7:320-2.

2. Nettleship E. Rare form of urticaria. Br Med J 1869;2:323-4.

3. Brockow K. Epidemiology, prognosis, and risk factors in mastocytosis. Immunol Allergy Clin North Am 2014;34:283-95.

4. Méni C, Bruneau J, GeorginLavialle S, et al. Paediatric mastocytosis: a systematic review of 1747 cases. $\mathrm{Br}$ J Dermatol 2015;172:642-51.

5. Neri I, Guareschi E, Guerrini V, Patrizi A. Familial teleangiectasia macularis eruptiva perstans. Pediatr Dermatol 2005;22:488-9.

6. Valent P, Akin C, Sperr WR, et al. Mast cell proliferative disorders: current view on variants recognized by the World Health Organization. Hematol Oncol Clin North Am 2003;17:1227-41.

7. Theoharides TC, Valent P, Akin C. Mast Cells, mastocytosis, and related disorders. N Engl J Med 2015;373:163-72.

8. Komi DEA, Rambasek T, Wöhrl S. Mastocytosis: from a molecular point of view. Clin Rev Alerg Immunol 2017;54:397-411.

9. Wolff K, Komar M, Petzelbauer P. Clinical and histopathological aspects of cutaneous mastocytosis. Leuk Res 2001;25:519-28.

10. Alvarez-Twose I, Vañó-Galván S, Sánchez-Muñoz L, et al. Increased serum baseline tryptase levels and extensive skin involvement are predictors for the severity of mast cell activation episodes in children with mastocytosis. 2012;67:813-21.

11. Lange M, Zawadzka A, Schrörs S, et al. The role of serum tryptase in the diagnosis and monitoring of pediatric mastocytosis: a singlecenter experience. Postepy Dermatol Alergol 2017;34:306-12.

12. Haneef NS, Ramachandra S, Metta AK, Srujana L. Chronic bullous disease of childhood with IgG predominance: What is the locus standi? Indian J Dermatol 2012;57:285-7.

13. Zone JJ, Egan CA, Taylor TB, Meyer LJ. IgA autoimmune disorders: development of a passive transfer mouse model. J Investig Dermatology Symp Proc 
2004;9:47-51.

14. Slavescu KC, Chiorean R, Danescu, $\mathrm{S}$, et al. Diffuse cutaneous bullous mastocytosis with IgM deposits at dermo-epidermal junction. J Cutan Pathol 2015;43:263-9.

15. Cardet JC, Akin C, Lee MJ. Mastocytosis: update on pharmacotherapy and future directions. Expert Opin Pharmacother 2013;14:2033-45.
16. Worobec AS. Treatment of systemic mast cell disorders. Hematol Oncol Clin North Am 2000;14:659-87.

17. Klaiber N, Kumar S, Irani AM. Mastocytosis in children. Curr Allery Asthma Rep 2017; 17:80.

18. Bulat V, Mihić LL, Situm M, et al. Most common clinical presentations of cutaneous mastocytosis. Acta Clin Croat 2009;48:59-64.

19. Cook EB, Stahl JL, Barney NP,
Graziano FM. Mechanisms of antihistamines and mast cell stabilizers in ocular allergic inflammation. Curr Drug Targets Inflamm Allergy 2002;1:167-80.

20. Belvisi MG. Regulation of inflammatory cell function by corticosteroids. Proc Am Thorac Soc 2004;1:207-14. 